\title{
Distortion correction for wide angle holographic projector
}

\author{
Jędrzej Szpygiel, Maksymilian Chlipała, Rafał Kukołowicz, Moncy Idicula, Tomasz Kozacki*
}

Faculty of Mechatronics, Warsaw University of Technology, 8 Św. A. Boboli St. 75, 02-525 Warsaw, Poland

Received October 15, 2021; accepted December 16, 2021; published December 31, 2021

\begin{abstract}
This letter presents a distortion correction method enabling a distortion minimized, large size image in a wide angle holographic projector. The technique applies numerical predistortion of an input image used for hologram generation. It is based on estimation of distortion coefficients by comparing optically a reconstructed poin test chart with the original one. Obtained experimental results prove that the technique allows reconstruction of high-quality image.
\end{abstract}

A lensless holographic projector built with a phase SLM (Spatial Light Modulator) enables the reconstruction of a high quality, aberration free image [1]. The main limitation of the display is a small projection angle, which is closely related to the pixel pitch of the used SLM. For an SLM with a pixel pitch size of $3.74 \mu \mathrm{m}$ the projection angle is $\pm 4.8^{\circ}$. Thus, it should be increased to display larger images.

In state-of-the art limited projection, the angle is addressed by two solutions, utilizing a spherical illumination module [2] or a $4 \mathrm{~F}$ imaging system [3, 4]. In the first technique the projection angle depends on the radius of curvature of the illuminating beam. The method is limited by the overlap of reconstructed images of higher diffraction orders. In the second approach, two lenses with different focal lengths demagnify the pixel pitch of the SLM. The limitation of the 4F method is the focal length ratio, as the demagnification increases the projector becomes bulkier.

Recently we have proposed a wide-angle holographic projector with a single lens and efficient hologram generation utilizing complex coding, and nonparaxial diffraction [5]. The characteristic feature of optical systems using a lens is the formation of aberrations. The most problematic and visible for a wide-view-angle holographic projector is the aberration of distortion, which needs to be compensated.

There are two main approaches of aberration correction: (i) using additional physical optical elements or a wavefront sensor and a deformable mirror and (ii) utilizing numerical correction by means of algorithms and digital image processing. The disadvantage of the first approach is its high cost, time consuming alignment and non-adaptivity. Numerical methods are attractive since the hologram is in most cases computer generated or there is an access to the complex object wavefront. Zehao He et

${ }^{*}$ E-mail: tomasz.kozacki@pw.edu.pl al. proposed a technique that detects distortion basing on the position of points regionally divided and corrects it by means of planar and phase divergence correction factors [6]. In the solution, the image is divided into subregions, for which correction factors are calculated individually, and correction is made by pre-calibrating images before projection. Another approach was proposed by Kaczorowski et al., in which the aberrations are corrected by means of a phase mask composed of 13 Zernike Polynomials [7]. The phase mask is uploaded onto the SLM, and its effectiveness is optimized by using two proposed algorithms - a hybrid genetic steepest descent and a heuristic variant of steepest descent. The mask maximizes the peak intensity of the point and minimizes the spread of pixels around the center, while imaging a point object. Zehao He et al. avoided the computational complexity of Zernike polynomials by implementing the angular spectrum algorithm and treating the image distortions as additional distribution superposed onto a computer-generated hologram [8]. In the work, the wavefront needed for eliminating the aberrations was acquired by a four-step phase shifting algorithm, which resulted in a reduced correction time.

Most solutions present in the literature require iterative algorithms of complex mathematic structure, which increase the correction time and computational requirements. The method proposed in this paper is based on a single pre-calibration step and pre-distorting the image in such a way that the distortion introduced by the system will be eliminated. The distortion coefficients are determined by detecting and comparing the position of projected test chart points to the desired ones. The radial and tangential distortions are considered. In this regards, the Fitzgibbons model is used, since it allows to describe much larger radial distortion with lower order terms. The assumed model of distortion can be presented by the following equations:

$$
\begin{aligned}
& x_{d}=x_{u}+x_{u} k r^{2}+q\left(r^{2}+2 x^{2}\right)+2 p x y \\
& y_{d}=y_{u}+y_{u} k r^{2}+2 q x y+p\left(r^{2}+2 y^{2}\right)
\end{aligned}
$$

where $x_{d}, y_{d}$ and $x_{u}, y_{u}$ are the distorted and undistorted coordinates respectively, $k, p$ and $q$ are the radial and tangential distortion coefficients. In order to measure image distortion, its coefficients are estimated by the 
displacement of a projected rectilinear grid of points. Next, the obtained data are used to pre-distort an image before projection to cancel the system's distortion. Since the distortion is the feature of the optical setup and does not depend on the projected image, only a single calibration step is needed. Firstly, the hologram of a test chart in a form of points array is displayed on the SLM. Then, by means of digital image processing the coordinates of points are detected. Since the ideal image is known, the desired spatial distribution of the points can be calculated for known projection parameters, such as projection distance, size of the SLM, camera pixel pitch, and wavelength. In order to calculate the distortion that influences the projected image, the ideal and detected points are compared and their corresponding coordinates are put into the following matrix equation:

$$
\left[\begin{array}{l}
k \\
p \\
q
\end{array}\right]=\left[\begin{array}{ccc}
x_{u} r^{2} & 2 x y & \left(r^{2}+2 x^{2}\right) \\
y_{u} r^{2} & \left(r^{2}+2 y^{2}\right) & 2 x y
\end{array}\right]^{+} \cdot\left[\begin{array}{l}
x_{d} \\
x_{d}
\end{array}\right],
$$

where “+” denotes a pseudo-inverse matrix, since the matrix with unknown coefficients is not a square one, a pseudo-inverse matrix has to be calculated. What is more, since the detected distorted points do not follow the exact mathematical model of a distortion, an approximation is necessary to avoid a contradictory system of equations. These calculations provide the best fitting radial and tangential distortion coefficients. In order to compensate the distortion of the optical setup a pre-distortion of the image before projection is needed, so the distortions cancel each other out. The input image is processed and projected by a holographic display system.

The proposed pre-distortion approach is applied in an image generation framework of wide-angle holographic projection display, which is presented in Fig. 1, to improve image quality. It employs laser source (lambda $=$ $632,8 \mathrm{~nm}$ ), plane wave illumination, $4 \mathrm{~K}$ phase only LCoS SLM (HOLOEYE Photonics AG, model GAEA-2, reflective phase only Liquid Crystal on Silicon microdisplay, resolution $4160 \times 2464,3.74 \mu \mathrm{m}$ pixel size) for hologram displaying, Fourier Transforming lens $\mathrm{L}_{\mathrm{p}}$ (focal length $F_{p}=35 \mathrm{~mm}$ ) for enlarging viewing angle, and rectangular spatial frequency filter to enable complex wave encoding. The lens ensures a wide projection angle $\alpha_{x}$, which depends on the size of a used SLM and optical power of the lens as $\alpha_{x}=\sin ^{-1}\left(B_{x} / F_{p}\right)$. For $F_{p}=35 \mathrm{~mm}$ and projection distance $z_{p}=700 \mathrm{~mm}$ image has the size 306 $\mathrm{mm} \times 161 \mathrm{~mm}$. The filter has two tasks: to decode the complex phase-coded information [9] and to pass only $1^{\text {st }}$ diffraction order of the SLM. In our system the SLM is tilted and information order is separated from the zero order by adding a spatial carrier frequency to the hologram.

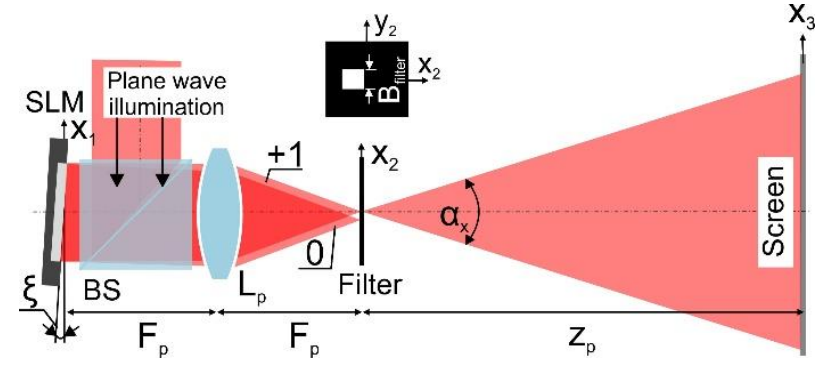

Fig. 1. Scheme of a wide-angle holographic projection display.

The diagram for the hologram generation algorithm is presented in Fig. 2. It consists of five steps: (i) predistortion of the image with calculated coefficients using Equation 3, (ii) nonparaxial propagation of an optical wavefield from the image plane $\left(x_{3}, y_{3}\right)$ to the filter plane with Angular Spectrum Compact Space Bandwidth Product method [10], (iii) band-limiting the signal in the frequency domain to one fourth of the system bandwidth, (iv) calculation of Fast Fourier Transform giving optical field in SLM plane $\left(x_{1}, y_{1}\right)$, and (v) generation of Double Phase Hologram [9]. The steps (iii) and (iv) are included for clear understanding of the algorithm only. The step (iv) does not have to be performed. In the algorithmic part of step (iii) there is an access to the Fourier Transform of the field at the filter plane.

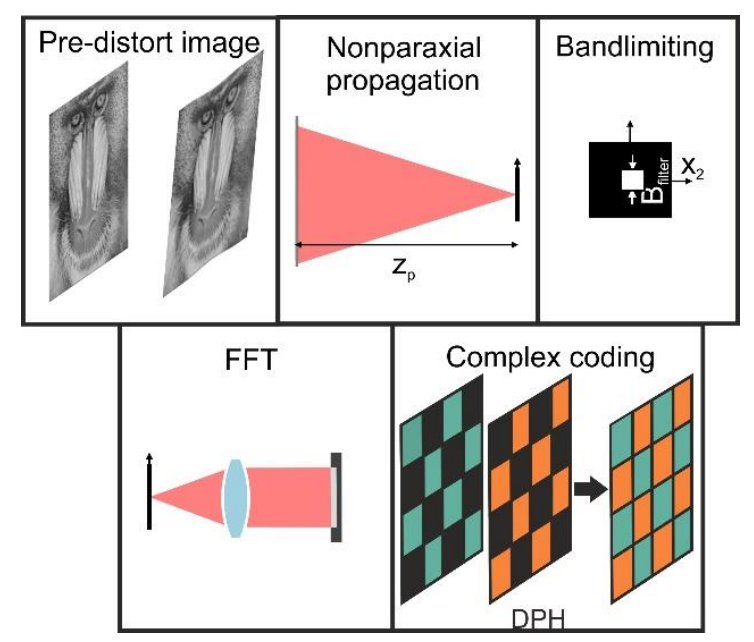

Fig. 2. Hologram generation algorithm.

To find the distortion coefficient of holographic display, the image of a test points chart consisting of 13 columns and 9 rows was generated and projected through the system at $7 \mathrm{~mm}$ and captured by a $4 \mathrm{~K}$ CCD camera. The detected coordinates were compared to the ideal ones and the distortion coefficients were calculated using Equation 3 , giving the following values:

$$
\begin{gathered}
k=5.514 \cdot 10^{-9} \\
p=-3.112 \cdot 10^{-6}, \quad q=-6.598 \cdot 10^{-6} .
\end{gathered}
$$


The test chart was then pre-distorted with the same distortion coefficients values but with the opposite signs. In Fig. 3 the distorted and corrected images of the test chart are superimposed and colored to visualize the distortion correction. Blue dots correspond to the distorted image and red ones to the corrected one.

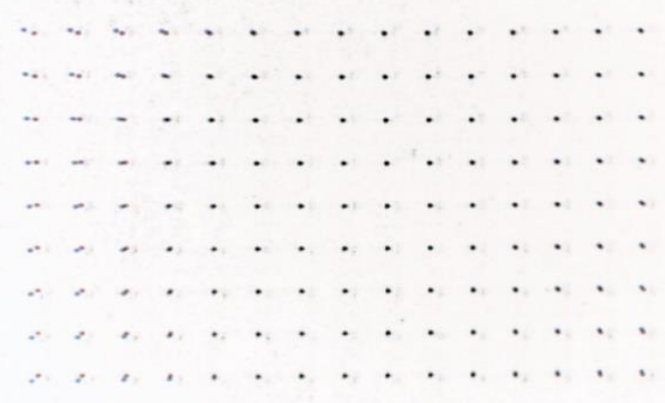

Fig. 3. Test chart before (blue) and after distortion correction (red).

The coordinates of the corrected chart were detected and compared to the ideal and distorted images. The quality of correction was also measured by comparing the mean displacement error of the distorted coordinates from the ideal to the corrected ones. The RMS error of displacement diminished significantly from 27 to 13 pixels, showing the range of correction.

The proposed method was investigated optically by reconstructing a 2D "Mandril" image. For this, two holograms were generated and reconstructed at distance $z_{p}$ $=700 \mathrm{~mm}$. The first hologram was calculated from the original image and the second from the pre-distorted image. Fig. 4 presents photos captured by the zoom lens camera. The obtained results show that the method allows for obtaining a high quality distortion corrected image.
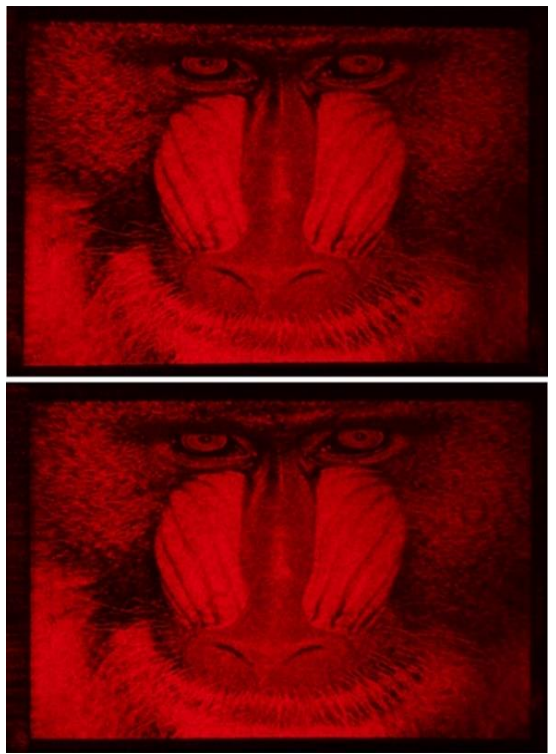

Fig. 4. Optically reconstructed "Mandril" image before (upper) and after distortion correction (lower).
In conclusion, this letter presents a distortion compensation method allowing us to reconstruct a distortion minimized, large-size image in a wide-angle holographic projector. The technique is based on single pre-calibration of the setup and pre-distorting the image. The pre-calibration is realized in two steps. First, optical reconstruction of the point array test chart is obtained in the display and acquired by a CCD. Next, the positions of points in space are numerically detected, compared with the reference ones and distortion coefficients $k, p, r$ are calculated. The calculated distortion coefficients are applied to pre-distort the image so the distortion introduced by the system will be cancelled out. Optical reconstructions prove that the proposed method allows for obtaining a high quality distortion corrected image.

Fundings: Politechnika Warszawska; Narodowe Centrum Nauki (UMO-2018/31/B/ST7/02980).

\section{References}

[1] M. Makowski, Experimental Aspects of Holographic Projection with a Liquid-Crystal-on-Silicon Spatial Light Modulator, in Holographic Materials and Optical Systems, M. Kumar, ed. (IntechOpen, 2019).

[2] H. Pang, A. Cao, W. Liu, L. Shi, Q. Deng, Appl. Opt. 58, 8713 (2019).

[3] Y. Qi, C. Chang, J. Xia, Opt. Express 24, 30368 (2016).

[4] E. Buckley, J. Display Technol. 99, 1 (2010).

[5] M. Chlipała, T. Kozacki, H. Yeom, J. Martinez-Carranza, R. Kukołowicz, J. Kim, J. Yang, J. Choi, J. Pi, C. Hwang, Opt. Lett. 46, 4956 (2021).

[6] Z. He, X. Sui, L. Cao, G. Jin, "Image-Distortion Correction Algorithm for Computer-Generated Holographic Display," 2018 IEEE 27th International Symposium on Industrial Electronics (ISIE), 1331 (2018).

[7] A. Kaczorowski, G.S. Gordon, A. Palani, S. Czerniawski, T.D. Wilkinson, J. Display Technol. 11(7), 596 (2015)

[8] Z. He, X. Sui, G. Jin, L. Cao, IEEE Trans. Industr. Inform. 15, 6162 (2019)

[9] O. Mendoza-Yero, G. Mínguez-Vega, J. Lancis, Opt. Lett. 39, 1740 (2014).

[10] T. Kozacki, K. Falaggis, Appl. Opt. 55, 5014 (2016). 